\title{
(6) OPEN ACCESS \\ The key actor: a qualitative study of patient participation in the handover process in Europe
}

Maria Flink, ${ }^{1,2}$ Gijs Hesselink, ${ }^{3}$ Loes Pijnenborg, ${ }^{4}$ Hub Wollersheim, ${ }^{3}$ Myrra Vernooij-Dassen, ${ }^{3,5,6}$ Ewa Dudzik-Urbaniak, ${ }^{7}$ Carola Orrego, ${ }^{8}$ Giulio Toccafondi, ${ }^{9}$ Lisette Schoonhoven, ${ }^{3,10}$ Petra J Gademan, ${ }^{11}$ Julie K Johnson, ${ }^{12}$ Gunnar Öhlén, ${ }^{13,14}$ Helen Hansagi, ${ }^{15}$ Mariann Olsson, ${ }^{1,2}$ Paul Barach, ${ }^{16,17}$ on behalf of the European HANDOVER Research Collaborative

For numbered affiliations see end of article.

Correspondence to Maria Flink, Department of Neurobiology, Care Sciences and Society, Karolinska Institutet, B44, Karolinska Sjukhuset Huddinge, Stockholm 14186, Sweden; maria.flink@ki.se

Accepted 25 August 2012 Published Online First 29 October 2012

\section{ABSTRACT}

Background: Patient safety experts have postulated that increasing patient participation in communications during patient handovers will improve the quality of patient transitions, and that this may reduce hospital readmissions. Choosing strategies that enhance patient safety through improved handovers requires better understanding of patient experiences and preferences for participation. Objective: The aim of this paper is to explore the patients' experiences and perspectives related to the handovers between their primary care providers and the inpatient hospital.

Methods: A qualitative secondary analysis was performed, based on individual and focus group patient interviews with 90 patients in five European countries.

Results: The analysis revealed three themes: patient positioning in the handover process; prerequisites for patient participation and patient preferences for the handover process. Patients' participation ranged from being the key actor, to sharing the responsibility with healthcare professional(s), to being passive participants. For active participation patients required both personal and social resources as well as prerequisites such as information and respect. Some patients preferred to be the key actor in charge; others preferred their healthcare professionals to be the key actors in the handover.

Conclusions: Patients' participation is related to the healthcare system, the activity of healthcare professionals and patients' capacity for participation. Patients prefer a handover process where the responsibility is clear and unambiguous. Healthcare organisations need a clear and well-considered system of responsibility for handover processes, that takes into account the individual patient's need of clarity, and support in relation to his/hers own recourses.

\section{INTRODUCTION}

Each transition of care, including the handover between the inpatient hospital and the patient's home and primary care setting is a potential threat to patient safety. ${ }^{1}$ Several studies have found that a suboptimal patient handover at hospital admission or discharge may lead to adverse events, with this contributing to suboptimal care, rehospitalisation and even death. ${ }^{1-5}$ Deficits in handover are often related to inadequate communication between healthcare professionals, with insufficient or unclear information exchanged between the hospital and primary care professionals. $^{3}$

Safe and effective transitions of care between care settings require healthcare professionals that give clear, unambiguous and understandable information to patients. ${ }^{6} 7$ Some handover communication involves not only the healthcare professionals transitioning the patient from one phase of care to the next, but also the patient. Improvements in hospital to primary care handovers that actively include the patient in the exchange of information between settings and healthcare professionals have been associated with reduced rehospitalisation, ${ }^{8} 9$ and faster delivery of information to primary healthcare professionals. ${ }^{3}$

While the knowledge about ways to improve the hospital to primary care handover continuum is increasing, there are few studies that have examined patient participation in handovers. The factors behind patient participation in the larger domain of healthcare decision-making have been studied. Patients differ in their participation in decision-making based on demographics, such as age ${ }^{10}$ health literacy; ${ }^{10}{ }^{11}$, national culture ${ }^{12}$ and expectations for participation. ${ }^{13}$ Improved knowledge about how patients experience their participation in 
handover processes between community and hospital care may help in finding optimal ways of empowering patients that will improve the quality and safety of handovers. This study aims to explore the experiences and perspectives of patients with chronic diseases in regards to their participation in handover communication between primary and secondary healthcare in five European countries.

\section{METHODS}

\section{Study design and settings}

This study is a secondary analysis of data from a crossnational, qualitative study of patient perspectives on their handovers, conducted at nine hospitals and their feeder primary healthcare systems in the Netherlands, Spain, Poland, Italy and Sweden. The settings were chosen to include different regional healthcare systems and hospitals of different sizes. The study was conducted as part of the European HANDOVER Project that researched handovers between primary and secondary care and examined the perspectives of a wide group of stakeholders in the transition of care between the inpatient hospital and the primary care and community setting (FP7-HEALTH-F2-2008-223409). ${ }^{14}$

\section{Primary study population}

Patients in the HANDOVER Project encompassed adults with a chronic disease (diabetes mellitus, chronic obstructive pulmonary disease (COPD), heart failure, asthma and/or poly-pharmacy) who were discharged to home directly from an inpatient hospital admission. Additional country-specific inclusion criteria are shown in table 1 . The general inclusion criteria were chosen to study patient handovers in both primary and secondary care settings. Patients were expected to have experienced several handovers. The population was chosen because handovers are critical for chronic and high-risk patients who require more frequent and complex transitions, ${ }^{6}$ and improving handovers for this group was thought to have a sizable impact on their quality of care. The country-specific criteria were used to recruit patients who had experienced handovers in different specialities and clinical settings. Purposive sampling was used to select patients with the chosen diagnoses, ages and gender. ${ }^{15}$

\section{Data collection}

Individual interviews were conducted with patients in their native language in 2009, and were done in person usually at the patients' home or at the hospital 3 to 4 weeks after discharge, sometimes with a family member present. In all countries, two members of the local research team conducted the interviews. All interviewers had experience with healthcare, either as researchers or as healthcare professionals, and were experienced interviewers or had attended a series of workshops on qualitative interviewing to ensure standardised methods.

Focus group interviews were performed in the patients' native language. Focus groups were led by a

\begin{tabular}{|c|c|c|c|c|}
\hline Country & $\begin{array}{l}\text { Individual } \\
\text { interviews } \\
(n=53)\end{array}$ & $\begin{array}{l}\text { Focus group } \\
\text { interviews } \\
(n=37)\end{array}$ & $\begin{array}{l}\text { Country specific inclusion } \\
\text { criteria }\end{array}$ & General inclusion criteria \\
\hline $\begin{array}{l}\text { The } \\
\text { Netherlands }\end{array}$ & $n=8$ & $n=7$ & $\begin{array}{l}\text { Patients admitted to internal } \\
\text { medicine, pulmonary diseases, } \\
\text { cardiology or (vascular) surgical } \\
\text { wards }\end{array}$ & $\begin{array}{l}>18 \text { years } \\
\text { Diagnosed with either diabetes } \\
\text { mellitus, chronic obstructive } \\
\text { pulmonary disease (COPD), }\end{array}$ \\
\hline Spain & $n=8$ & $n=3$ & $\begin{array}{l}\text { Patients belonging to cultural } \\
\text { minority groups with } \\
\text { limited health literacy (capacity to } \\
\text { read, write, and understand } \\
\text { healthcare information) }\end{array}$ & $\begin{array}{l}\text { heart failure, asthma or/and } \\
\text { poly-pharmacy ( }>6 \text { drugs) } \\
\text { Discharged to home or nursing } \\
\text { home (under responsibility of } \\
\text { primary/community care) }\end{array}$ \\
\hline Poland & $\mathrm{n}=23$ & $\mathrm{n}=10$ & Patients $\geq 60$ of age & \\
\hline Italy & $\mathrm{n}=5$ & $\mathrm{n}=9$ & $\begin{array}{l}\text { Patients admitted to emergency } \\
\text { ward }\end{array}$ & \\
\hline Sweden & $n=9$ & $n=8$ & $\begin{array}{l}\text { Patients admitted to emergency } \\
\text { ward via emergency room. If } \\
\text { living at a nursing home, only } \\
\text { within a geographically specified } \\
\text { area }\end{array}$ & \\
\hline
\end{tabular}

Number of participants by country and inclusion criteria. 
trained moderator, and had one or two observers who made field notes and added question prompts. All interviews were audio-taped and transcribed verbatim in the local language, according to a jointly decided standardised format.

Both individual and focus groups interviews used a semistructured interview guide, developed in English by the HANDOVER Project researchers and translated into the local language of the research groups. ${ }^{16}$ The interview guide covered these areas of interest:

- Experience with recent handovers (appreciative/ problematic situations and consequences)

- Perceptions about handovers in general (ie, experiences, attitudes, methods, tools, barriers, facilitators)

- Perceptions about provider/patient tasks, roles and responsibilities

- Suggestions for improving patient handovers

The guide was piloted in each country and when necessary, adjusted for local conditions and needs (available upon request).

A quality assurance $\operatorname{protocol}^{17}$ based on $B M J$ criteria $^{18}$ and on criteria presented by Tong $e t a l^{15}$ was developed (by MVD and JKJ) and used to ensure trustworthiness throughout the data collection and data analyses. Requirements for informed consent and other ethical and legal requirements for research using patient information were fulfilled at all study sites.

\section{Sample for secondary analysis}

For the secondary analysis data were extracted from patient interviews in the five countries participating in the HANDOVER Project. The sample consisted of 90 patients, 53 individually interviewed $(55 \%$ of the overall sample of patients interviewed individually for the original studies conducted as part of the HANDOVER Project) and 37 interviewed in focus groups (100\% of the overall sample of patients interviewed in groups).
The distribution across countries is shown in table 1 . Where the gender distribution of the primary sample was reported, it included approximately equal numbers of male and female patients.

\section{Data analysis}

The analysis consisted of two main parts: analysis of interviews at the national level, and a secondary analysis ${ }^{19}$ of the cross-national data (see figure 1).

\section{Analysis at national level}

Two interviews from each country were translated into English, and coded inductively using a modified grounded theory approach of Corbin and Strauss. ${ }^{20} \mathrm{~A}$ qualitative data analysis software (Atlas.ti) was used to facilitate storing, coding and indexing of the data. ${ }^{21}$ The researchers created a codebook based on codes generated in each country. The codebook consisted of the code and an operational definition, both of which were agreed upon during regularly scheduled conferencecalls and face to face meetings, and was used for analysis of both individual and focus group interviews. Two researchers in each country analysed their country's data in parallel with the data collection, and continued to collect data until conceptual saturation was reached and no new codes or categories were generated. ${ }^{20}$ Local reports were written in English by one or two of the researchers in each country and used for compiled analyses for the European HANDOVER Project studies. These studies focused on barriers and facilitators to effective handovers; patient roles and responsibilities; and patient-centredness and participation culture. ${ }^{1622} 23$

\section{Secondary analysis}

The local reports, including quotes from the individual interviews and focus groups, were used for the

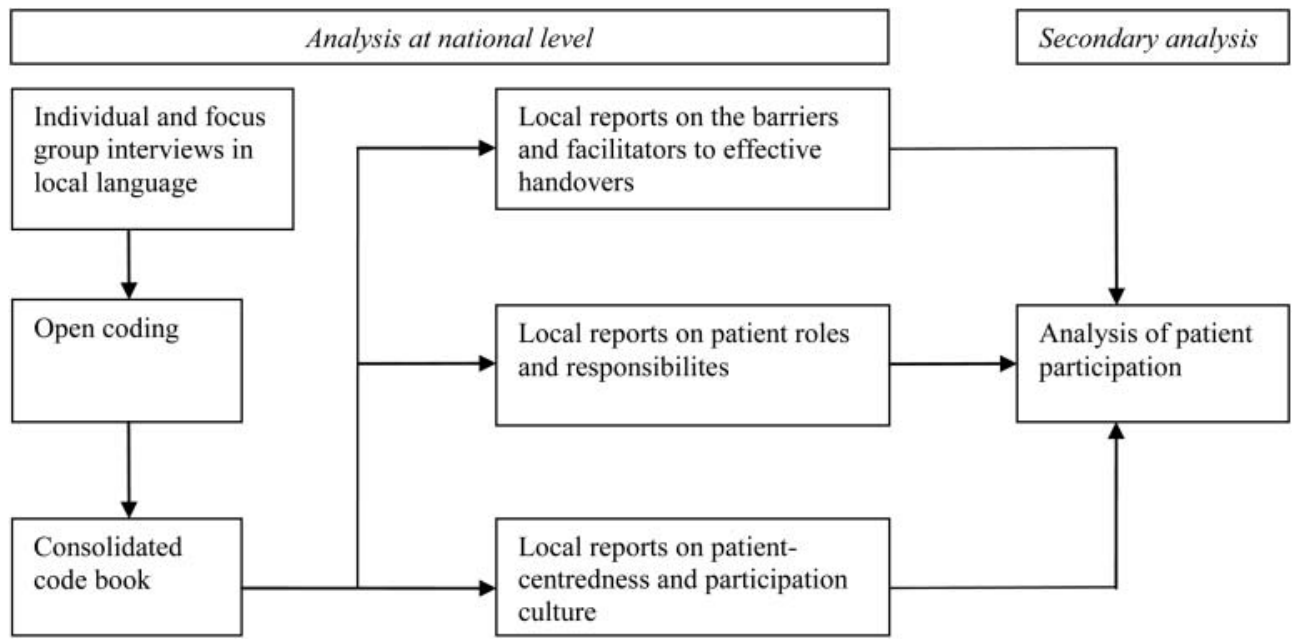

Figure 1 Procedure-from data collection to the current secondary analysis. 
secondary analysis, which focused on patients' perspective on their own participation in handovers. Thus, this secondary analysis seeks to answer a new research question, ${ }^{19}$ using data that was already collected.

Local reports were analysed using a qualitative inductive content analysis as described by Hsieh and Shannon. ${ }^{24}$ Two authors (MF, MO) coded the texts in open coding and three authors (MF, MO, GH) sorted the codes into categories and themes. Validity checking involved all authors of the local reports, who reviewed the findings to ensure they were consistent with the original interview data.

\section{RESULTS}

The data analysis resulted in eight categories, from which three themes were developed: (1) patient positioning in the handover process; (2) prerequisites for patient participation; and (3) patient preferences for the handover process (see table 2).

\section{Patient positioning in the handover process}

Three types of patient experiences form the categories under this theme: (a) patients as the key actors in the handover process; (b) patients sharing the responsibility for the handover with healthcare professionals; and, (c) healthcare professionals functioning as the key actors in the handover process.

\section{Table 2 Categories and themes}

Categories Themes

1. Patients as the key actors in the handover process

2. Patients sharing the responsibility for the handover with healthcare professionals

3. Healthcare professionals functioning as the key actors in the handover process

4. Actions required for patients to be key actors

5. Resources and discipline required to be the key actor

6. Facilitators for and barriers to patient participation

7. Patient preferences for being the key actors

8. Patient preferences regarding healthcare professionals serving as the key actors

\section{Patients as the key actors in the handover process}

Patients assumed the responsibility for the handover communication, including establishing contact with the next care unit as well as the responsibility for collecting, storing and handing over essential information for managing their care, such as medication lists or discharge notes. For example, when a healthcare professional in the hospital setting in Italy wrote the discharge note to transition the patient to the primary care setting-this discharge note was given to the patient for delivery to the general practitioner. The key actor positioning was most explicitly stated by the geriatric patients in Poland, who noted they needed to take responsibility in organising the handovers as well as being couriers.

Patients who functioned as key actors perceived their active involvement was required for an effective handover and to ensure continuity of care. These patients had either learned from past experiences that little or no information was transferred unless they did it themselves, or perceived that healthcare professionals expected them to assume the initiative and be active during their handovers.

Patient, Poland: "Well, we all know by now that it [general practitioner - hospital communication] doesn't exist. You want a referral-then you get it. Then I arrange my admission-neither my general practitioner nor hospital physician care ..."

Being the key actor could cause some patients to blame themselves for not fulfilling the task of facilitating the handover when information was lacking between care settings.

Patient, Italy: "It was our fault. We forgot to contact the general practitioner during the hospitalisation."

\section{Patients sharing the responsibility for the handover with healthcare professionals}

Examples of patients sharing responsibility for the handover with healthcare professionals were found in Sweden for patients who were admitted for acute conditions. These patients participated in sharing essential or specific information for their care transition, which complemented the handover communication conducted by the healthcare professional. For example, during hospital admissions the patients informed healthcare professionals about their medications and previous care episodes, and at discharge, they informed the healthcare professionals about the name or address of their general practitioner to ensure that the correct primary care professional received the handover information. Healthcare professionals actively encouraged patients to share the responsibility for communication, such as ensuring that proper information was shared between the hospital and 
community/primary care settings and asked of the patients to assume responsibility for their own health.

Patient, Sweden: "They used to say, please call the advanced home care services, just for safety reasons, and tell them you're back home."

\section{Healthcare professionals functioning as the key actors in the handover process}

Some patients viewed the healthcare professionals as the key actors in the handover process. These patients assumed that handovers are performed by healthcare professionals, focusing on verbal and/or written communication, electronic mail or medical records data shared between the care settings. This more passive positioning in the handover was found among the Dutch patients. Some hospitals in the Netherlands had a dedicated 'transfer nurse' to manage handovers to the primary care setting after discharge.

Patient, Netherlands: "Yes, I indeed think that if the hospital takes responsibility to discharge patients because they think they can manage outside the hospital, they also have to take that responsibility and arrange an alternative solution if it's not possible."

\section{Prerequisites for patient participation}

This theme had three categories: (a) actions required for patients to be key actors; (b) resources and discipline required to be the key actor; and, (c) facilitators for and barriers to patient participation.

\section{Actions required for patients to be key actors}

To be able to function as key actors, patients had to assume the responsibility, that is, take initiatives and ask the healthcare professionals questions to get the needed information.

Patient, Spain: "In order to have more information, it is important to ask ... and sometimes, you ask a question, and they answer "Well, I don't know...Wait please, I will ask somebody" ... and they don't tell you anything."

In addition, patients had to be explicit and sometimes be assertive in their communication with the healthcare professionals to help the handover move forward.

\section{Resources and discipline required to be the key actor}

Patients acknowledged the importance and the potential of having own resources, and noted they investigated their social network to find the best healthcare professional available. Patients also used their available family and contact resources. Family members sometimes replaced the patient in taking responsibility for conveying handover information and acting as medical secretaries or information conduits.

Patients with limited personal resources or with low health literacy had difficulties understanding the received information and sharing the information with the healthcare professionals. This limited their participation in the handover communication.

Patient, Spain: "What can I say? The main thing was that I could not speak in Spanish so I can't express many things, so that is the problem."

In order to function as the key actors in the handover, geriatric patients in Poland had to be disciplined in organising and transferring medical documents as they were the main repository of patient documentation. In this model, lack of discipline and willingness to systematically collect relevant documents were barriers to effective handovers.

Patient, Poland: "If the patient does not want and would not comply, the best doctor would not (be able to) help him."

\section{Facilitators for and barriers to patient participation}

Patients reported on several communication facilitators and barriers related to their participation. Patients perceived a positive climate for communication, based on mutual respect, in an open atmosphere and on a personal level, between them and the healthcare professionals as an enabling factor for participation in the handover. Accordingly, a negative climate for communication involved healthcare professionals neglecting patients' individual needs, or being too busy to communicate with patients.

Lack of information was the main barrier to participation during the handover process. Patients perceived a gap between the information they received and the information they actually needed for continuous care. Information gaps often concerned medication information; when patients were discharged with unclear or insufficient information on how best to handle their medications or without a new and updated medication list they could not participate actively in follow-up. Finally, the patients expressed the need for a dedicated discharge encounter in which they would be given all the information needed that could help them improve their postdischarge care.

Patient, Italy: "I go back home with a bag of drugs and trust me that this was a mess I could not sort out ... They didn't tell us that there could be a risk of depression. I had a medical discharge report, they have been really good for God's sake, but they did not explain to us enough."

\section{Patient preferences for the handover process}

There are two categories under this theme: (a) patient preferences for being the key actors; and (b) patient 
preferences regarding healthcare professionals serving as the key actors.

\section{Patient preferences for being the key actors}

The preference for being key actors in the handover process was expressed by Swedish patients undergoing acute admissions, by geriatric patients in Poland, and by patients in the Netherlands. These patients stressed the importance of patients in contributing to an effective handover. For example, Swedish patients noted that when they assumed responsibility for the handover, communication worked better. It also empowered them and gave them a sense of control over the handover process. Patients also found the opportunity to look up and ascertain the accuracy of the information being transferred an advantage.

Focus group, Netherlands

"Patient A: You receive the letter and you deliver it to the doctor, and then the general practitioner will visit to see how you are doing, so, this is very satisfying for me, yes.

Interviewer: So you are satisfied with this, that the information is routed via you?

Patient B: I find it an advantage when it is routed via the patient.

Patient A: Yes, and it's also true that you are certain the information is coming across."

\section{Patient preferences regarding healthcare professionals serving as the key actors}

Patients who indicated a preference for healthcare professionals to be the key actors in the handover perceived handovers more effective when healthcare professionals were actively involved. These patients felt that professionals should be fully responsible for the handover, and reported feeling frustrated when urged to take responsibility for the handover and wanted a passive role in their care. Some patients mentioned a transfer nurse as the preferred key actor for handovers.

Relative, Sweden: "They should have somebody who always gets in touch with the nursing home. Someone responsible, that can take care of all contacts."

One subgroup, patients with low health literacy, did not express any preferences regarding participating or not participating in the handover process.

\section{DISCUSSION}

Three themes of importance for patient participation were revealed in the study: patient positioning in the handover process, prerequisites for patient participation and patient preferences for the handover processes. This study does not make comparisons across countries. Instead the aim was to study patient participation in handover processes with different characteristics, in various care settings to explore patients' perspectives more indepth. The findings demonstrate that patients' positioning ranged from being the key actor, sharing the responsibility with healthcare professionals to being passive.

Patients' positioning seems to respond to the handover system in an elastic relation and are modulated by their perceptions of the healthcare professionals actions. In systems with less active engagement of healthcare professionals, patients assumed a more active position, while in systems with active engagement, particularly by dedicated professionals like transfer nurses, patients assumed a more passive position. Other studies have found that patient participation differs depending on the settings, patient and physician attributes, ${ }^{10} 2526$ and the experiences of patient participation in the handover processes mirror these findings. Due to the fact that our study was a secondary analysis of the data, we cannot be sure to which degree the positioning continuum was a result of the patient characteristic or reflects the characteristics of the participating nations' healthcare systems.

The passive role of some patients in the handover may be a consequence of these individuals lacking information or instructions from healthcare professionals that would allow them to actively participate, or a lack of personal resources, capabilities or discipline. To be able to participate actively, patients required certain resources and prerequisites (eg, social network, health literacy and clear information/instructions), as well as being treated with respect. Two recent reviews on patient participation found positive outcomes of patient participation include better interaction between patients and healthcare professionals, and enhanced patient safety. ${ }^{1027}$ The findings of this study thus raise the question whether the quality of handovers is reduced when patients are passive participants, because they may lack the prerequisites for active participation.

Many patients did not state a preference for shared responsibility, as one might have expected, but preferred that either the healthcare professionals or the patient functioned as the key actor. We have not found any earlier studies on patient preferences regarding assuming handover responsibility. Comparing our findings to studies on patient participation in decision-making ${ }^{11} 28$ reveals that patients choose to be passive participants when their involvement may have a negative affect on the outcome of the decision, when decisions are complex, and, when patients were severely ill. ${ }^{10}{ }^{11}$ Patients with cancer preferred a shared or an active role. ${ }^{28}$ Patients' preference for healthcare professionals to be the key actors in our study may be explained by 
their chronic disease status, and the requirement for sharing complex medical information, as well as in the patients' statements that a handover process with clear responsibility was most effective. Because patients in the primary interviews were not explicitly asked about their interest in shared responsibility, we cannot exclude that some patients' desire shared responsibility. However, our findings suggest that patients in our analysis appeared to prefer clarity about who is responsible for the handover, irrespective of whether the patient or the health professionals function as the key actor.

The study has several limitations. First, the initial translations of the interviews were conducted in the respective countries by the researchers themselves, and not by professional translators, and the secondary analysis was performed on the English text by Swedish and Dutch researchers, raising concerns about the potential for linguistic misinterpretation. This risk was reduced by having both the primary researchers and the individuals who conducted the secondary analysis actively involved in discussions about the study aim and methods, by having all steps in data collection and analysis monitored using a quality assurance programme,${ }^{17}$ and by ensuring that the authors of the local reports have read and confirmed the accuracy of data from the secondary analysis. Also, the secondary analysis was not performed directly on the original data but on data already selected for local reports by researchers and it was not possible to conduct a validity check with the original patients interviewed. Third, patient-specific information on age and the distribution of the chronic conditions is not known for the sample for secondary analysis. The primary study population in the HANDOVER-study included a representative distribution by age, gender and diagnoses. ${ }^{16} 2223$ Finally, the population was restricted to adult patients with chronic conditions, which may limit the ability to transfer the findings across all handovers.

The methodological limitations with secondary analysis have been well described by Thorne. ${ }^{19}$ A key issue lies in the distance between the original data source-the patients-and the secondary question about patient participation. This question was however a natural extension from the primary research questions of the HANDOVER-study.

\section{CONCLUSIONS}

This study, despite its limitations, increases our knowledge of the preferences of patients for participating in the handover between the inpatient and the primary care setting. Patient participation in handovers between primary and hospital care is related to the healthcare system, the activity of healthcare professionals and the patients themselves. The ability to participate and take an active positioning requires patients' personal and social resources, prerequisites such as personal and clear information and respectful treatment by healthcare professionals. Patients prefer a handover process where the responsibility for the handover communication is clear and unambiguous, that is, a system that ensures them in transparent manner there is continuity of their care. This is an important finding for efforts to improve patient handovers to create and sustain greater reliability, transparency and consistency.

Future improvements of the patient handover will require the healthcare organisations to develop a clear and well-considered system of assigning responsibility for this process. Regardless of the system chosen, the individual patient's need of clarity and a level of support that is tailored to his/her own resources and ability to participate in the handover must be taken into account. Future development and research is needed to find out how a shared responsibility could look like in practice and be unambiguous for the patients. Such knowledge can help enhance safe patient transitions between the hospital and the patient's home.

\section{Author affiliations}

${ }^{1}$ Department of Neurobiology, Care Sciences and Society, Karolinska Institutet, Stockholm, Sweden

${ }^{2}$ Department of Social Work, Karolinska University Hospital, Stockholm, Sweden

${ }^{3}$ Scientific Institute for Quality of Healthcare (IQ healthcare), Radboud University Nijmegen Medical Centre, Nijmegen, The Netherlands

${ }^{4}$ Department on Quality and Safety, St Antonius Hospital, and Department on Quality and Safety, St Antonius Hospital, Nieuwegein, The Netherlands

${ }^{5}$ Department of Primary Care, Radboud University Nijmegen Medical Centre, Nijmegen, The Netherlands

${ }^{6}$ Kalorama Foundation, Radboud University Nijmegen Medical Centre, Nijmegen, The Netherlands

${ }^{7}$ National Center for Quality Assessment in Health Care, Krakow, Poland

${ }^{8}$ Avedis Donabedian Research Institute (FAD), Universitat Autònoma de

Barcelona and CIBER Epidemiology and Public Health (CIBERESP), Barcelona,

Spain

${ }^{9}$ Clinical Risk Management and Patient Safety Centre, Tuscany Region,

Florence, FI, Italy

${ }^{10}$ Faculty of Health Sciences, University of Southampton, Southampton, UK

${ }^{11}$ Primary Health Care, Utrecht Area, Utrecht, The Netherlands

${ }^{12}$ Centre for Clinical Governance Research, University of New South Wales, Sydney, Australia

${ }^{13}$ Department of Clinical Science, Intervention and Technology, Karolinska Institutet, Stockholm, Sweden

${ }^{14}$ Quality and Patient Safety, Karolinska University Hospital, Stockholm, Sweden

${ }^{15}$ Department of Clinical Neuroscience, Karolinska Institutet, Stockholm,

Sweden

${ }^{16}$ Patient Safety Center, University Medical Center Utrecht, Utrecht, The Netherlands

${ }^{17}$ Department of Health Studies, University of Stavanger, Stavanger, Norway

Acknowledgements We would like to thank all participating patients for sharing their valuable experiences.

Collaborators The European HANDOVER Research Collaborative consists of: Venneri F, Albolino S, Molisso A, Toccafondi G (Azienda Sanitaria Firenze, Florence, Italy), Barach P, Gademan P, Göbel B, Johnson J, Kalkman C, Pijnenborg L (Patient Safety Center, University Medical Center Utrecht, 
Utrecht, The Netherlands), Wollersheim H, Hesselink G, Schoonhoven L, Vernooij-Dassen M, Zegers M (Scientific Institute for Quality of Healthcare, Radboud University Nijmegen Medical Centre, Nijmegen, The Netherlands), Boshuizen E, Drachsler H, Kicken W, van der Klink M, Stoyanov S (Centre for Learning Sciences and Technologies, Open University, Heerlen, the Netherlands), Kutryba B, Dudzik-Urbaniak E, Kalinowski M, Kutaj-Wasikowska H (National Center for Quality Assessment in Health Care, Krakow, Poland), Suñol R, Groene 0, Orrego C (Avedis Donabedian Institute, Universidad Autónoma de Barcelona, Barcelona, Spain), Öhlén G, Airosa F, Bergenbrant S, Flink M, Hansagi H, Olsson M (Karolinska University Hospital, Stockholm, Sweden), Lilford R, Chen Y-F, Novielli N, Manaseki-Holland S (University of Birmingham, Birmingham, UK).

Contributors Study conception and design: MF, LP, HW, MVD, CO, LS, JKJ, GO, HH, MO and PB. Acquisition of data: MF, GH, LP, HW, EDU, CO, GT, PG and MO. Analysis at national level- the local reports: MF, GH, LP, EDU, CO, GT, PG and MO. Secondary analysis: MF, GH, MVD, MO. Drafting and/or revising the article: MF, GH, LP, HW, MVD, EDU, CO, GT, LS, PG, JKJ, GO, $\mathrm{HH}, \mathrm{MO}$ and $\mathrm{PB}$.

Funding This study was supported by a grant from the European Union, the Framework Programme of the European Commission (FP7-HEALTH-F2-2008223409). The study sponsor had no role in the study design; collection, analysis, and interpretation of the data; or in the writing of the article and decision to submit the article for publication.

Competing interests None.

Ethics approval Ethical review boards in the Netherlands, Spain, Poland, Italy and Sweden

Provenance and peer review Not commissioned; externally peer reviewed.

Data sharing statement Data are available on request from the corresponding author.

\section{REFERENCES}

1. Forster AJ, Clark HD, Menard A, et al. Adverse events among medical patients after discharge from hospital. Can Med Assoc J 2004;170:345-9.

2. Forster AJ, Murff HJ, Peterson JF, et al. The incidence and severity of adverse events affecting patients after discharge from the hospital. Ann Intern Med 2003;138:161-7.

3. Kripalani S, LeFevre F, Phillips CO, et al. Deficits in communication and information transfer between hospital-based and primary care physicians-Implications for patient safety and continuity of care. JAMA 2007;297:831-41.

4. Moore C, Wisnivesky J, Williams S, et al. Medical errors related to discontinuity of care from an inpatient to an outpatient setting. J Gen Intern Med 2003;18:646-51.

5. Tam VC, Knowles SR, Cornish PL, et al. Frequency, type and clinical importance of medication history errors at admission to hospital: a systematic review. Can Med Assoc J 2005;173:510-15.

6. Halasyamani L, Kripalani S, Coleman E, et al. Transition of care for hospitalized elderly patients-Development of a discharge checklist for hospitalists. J Hosp Med 2006;1:354-60.

7. Johnson A, Sandford J. Written and verbal information versus verba information only for patients being discharged from acute hospital settings to home: systematic review. Health Educ Res 2005;20:423-9.
8. Coleman EA, Parry C, Chalmers S, et al. The care transitions intervention-Results of a randomized controlled trial. Arch Intern Med 2006;166:1822-8.

9. Jack BW, Chetty VK, Anthony D, et al. A reengineered hospital discharge program to decrease rehospitalization. A randomized trial. Ann Intern Med 2009;150:178-88.

10. Davis RE, Jacklin R, Sevdalis N, et al. Patient involvement in patient safety: what factors influence patient participation and engagement? Health Expect 2007;10:259-67.

11. Say R, Murtagh M, Thomson R. Patients' preference for involvement in medical decision making: A narrative review. Patient Educ Couns 2006;60:102-14.

12. Meeuwesen L, van den Brink-Muinen A, Hofstede G. Can dimensions of national culture predict cross-national differences in medical communication? Patient Educ Couns 2009;75:58-66.

13. Coulter A, Jenkinson C. European patients' views on the responsiveness of health systems and healthcare providers. Eur $J$ Public Health 2005;15:355-60.

14. Improving the continuity of patient care through identification and implementation of novel patient handover processes in Europe. http://www.handover.eu (accessed 21 Oct 2011).

15. Tong A, Sainsbury $P$, Craig J. Consolidated criteria for reporting qualitative research (COREQ): a 32-item checklist for interviews and focus groups. Int J Qual Health Care 2007;19:349-57.

16. Hansagi $\mathrm{H}$, Olsson M, Glas S, et al. Report on the barriers and facilitators to effective handovers in the social, linguistic and technological context in which they take place. Utrecht,

The Netherlands: Utrecht Medical Center, for the European Union, 2010.

17. Johnson J, Barach P, Vernooij-Dassen M. A standardized approach for conducting qualitative research across multiple countries. BMJ Quality and Safety 2012.

18. Mays N, Pope C. Qualitative research in health care. Assessing quality in qualitative research. BMJ 2000;320:50-2.

19. Thorne S. Ethical and representational issues in qualitative secondary analysis. Qual Health Res 1998;8:547-55.

20. Corbin J, Strauss A. Grounded theory research: Procedures, canons, and evaluative criteria. Qual Sociol 1990;13:3-21.

21. ATLAS.ti Scientific Software Development Company, GmbH, Berlin, Germany. http://www.atlasti.com/ (accessed 21 Mar 2011).

22. Barach $\mathrm{P}$, Gademan $\mathrm{P}$, Kalkman $\mathrm{C}$, et al. Report on how variations in handover processes lead to near misses and adverse outcomes, and identifying standardized elements of communication. Utrecht, The Netherlands: Utrecht Medical Center, for the European Union, 2010.

23. Hesselink G, Wollersheim $\mathrm{H}$, Barach $\mathrm{P}$, et al. Report on challenges in patient care and the factors that influence change in practice. Utrecht, The Netherlands: Utrecht Medical Center, for the European Union, 2010.

24. Hsieh HF, Shannon SE. Three approaches to qualitative content analysis. Qual Health Res 2005;15:1277-88.

25. Street RL Jr, Krupat E, Bell RA, et al. Beliefs about control in the physician-patient relationship: effect on communication in medical encounters. J Gen Intern Med 2003;18:609-16.

26. Street RL Jr, Gordon HS, Ward MM, et al. Patient participation in medical encounters: why some patients are more involved than others. Med Care 2005;43:960-9.

27. Longtin $\mathrm{Y}, \mathrm{Sax} \mathrm{H}$, Leape LL, et al. Patient participation: current knowledge and applicability to patient safety. Mayo Clin Proc 2010;85:53-62.

28. Tariman JD, Berry DL, Cochrane B, et al. Preferred and actual participation roles during health care decision making in persons with cancer: a systematic review. Ann Oncol 2010;21: 1145-51. 